\title{
Influence mechanism of running sportswear fatigue based on BP neural network
}

\author{
Xiaoli Liu ${ }^{1}$ and Zhibin $\mathrm{Li}^{2^{*}}$
}

\footnotetext{
* Correspondence: jasmine_1974@ 126.com

${ }^{2}$ College of Automation Engineering, Shanghai University of Electric Power, Shanghai 200090, China

Full list of author information is available at the end of the article
}

\begin{abstract}
The fatigue of running sportswear is reflected in the fatigue caused by the tightness of the tights on the skin surface of the limbs, trunk, and other parts during long-term running sports. However, the current research on the fatigue of running sportswear is not deep enough. Therefore, the purpose of this study is to study the mechanism of the fatigue of running sportswear based on BP neural network. This article first takes sportswear as the starting point and uses the surface myoelectricity index as a physiological quantity as a means to combine clothing with sports medicine and sports physiology, breaking the traditional shackles of subjective assessment of fatigue, and giving play to the advantages of interdisciplinary to expand the new direction of the apparel industry and, secondly, use muscle fatigue evaluation method to analyze the muscles of the lower leg under the pressure of sportswear to analyze the strength of the EMG signal and the number of participating sports units and the frequency of discharge synchronization. Experimental data shows that the AUC is 0.756 when wearing sports tights, the sensitivity and specificity are $72 \%$ and $19 \%$, and the accuracy is $65 \%$. The experimental results show that clothing pressure affects the fatigue of running sportswear based on BP neural network.
\end{abstract}

Keywords: BP neural network, Fatigue of running sportswear, Muscle fatigue evaluation, Eigenvalue of fatigue, BP neural network model

\section{Introduction}

\subsection{Background and significance}

With the development in recent years, the BP neural network model architecture has matured. The feature dimension of the data set determines the number of input layer neurons of the BP neural network, and the initial weight and threshold determine the classification accuracy rate. Therefore, the improvement work for BP neural network is mainly focused on the selection of data features, and the optimization of the weights and thresholds of the network, which is also in line with the current situation of the increasing size of the data and the characteristics of the initialization weights and thresholds of the network are uncertain. Due to the different participation in sports, the muscle fibers and exercise intensity will be different. In addition, the test equipment

(c) The Author(s). 2021 Open Access This article is licensed under a Creative Commons Attribution 4.0 International License, which permits use, sharing, adaptation, distribution and reproduction in any medium or format, as long as you give appropriate credit to the original author(s) and the source, provide a link to the Creative Commons licence, and indicate if changes were made. The images or other third party material in this article are included in the article's Creative Commons licence, unless indicated otherwise in a credit line to the material. If material is not included in the article's Creative Commons licence and your intended use is not permitted by statutory regulation or exceeds the permitted use, you will need to obtain permission directly from the copyright holder. To view a copy of this licence, visit http://creativecommons.org/licenses/by/4.0/. 
tests the difference the indexes make; there is no unified evaluation index and evaluation method for the evaluation of dress fatigue.

BP neural network and its variant models have been widely used in agricultural production, image recognition classification, and engineering control, including medical and health fields, and become a widely used algorithm in artificial neural networks [1, 2]. At the same time, the training suit aims to provide athletes with a scientific basis to improve their ability to resist muscle fatigue and improve competition performance and to promote the development of sports in China. It also provides new ideas for the design and development of special functional training suits and the development of tight-fitting sports equipment [3, 4].

\subsection{Related work}

At present, the research on the fatigue of running sportswear is not deep enough, but many scholars have done relevant research. Giandolini M's scientific experiments on running mainly consider the fatigue caused by horizontal running. However, the degree and cause of fatigue depend on the exercise considered, especially the main contraction types, which vary between horizontal exercise, uphill exercise, and downhill exercise. The purpose of this review is to comprehensively summarize the neurophysiological and biomechanical changes caused by fatigue during graded running. When comparing a long hill run (i.e., a combination of uphill and downhill running) with horizontal running, he found that the general shape of the neuromuscular fatigue-exercise duration curve and the flexors of the knee extensor and plantar fatigue are similar. The biomechanical results are also relatively comparable, suggesting that duration, rather than changes in altitude, will affect neuromuscular function and mode of operation. However, his research direction is too broad and does not involve the relevant factors of dress fatigue and he does not particularly focus on data analysis [5]. Based on the secondary development of the FLUKE 411D laser ranging module, Wu B proposed a general implementation method for laser ranging of micro UAVs. The micro UAV DJI Phantom1 completed the airborne dynamic laser ranging experiment. Through data processing based on the method based on least squares and BP neural network, the measurement error obtained based on the method based on BP neural network is $4.35 \%$, much lower than $6.69 \%$ based on least squares. This means that the method based on BP neural network can significantly improve the measurement accuracy. Therefore, the airborne laser ranging error compensation method based on BP neural network is effectively verified [6]. Jidong Wang's research on wind power forecasting mainly focuses on point forecasting, which makes it difficult to predict its uncertainty, resulting in less application in practice. A wind power range prediction model based on BP neural network multi-output characteristics is established, and an optimization criterion based on prediction interval information is proposed. The simulation results of the example show that the proposed wind power range prediction model can effectively predict the output power interval and provide the basis for decision-making for grid dispatchers [7]. Tongle $\mathrm{X}$ has developed and demonstrated a new method to deal with the difficulties of spectroscopic analysis in prompt gamma neutron activation analysis (PGNAA). It includes the use of BP neural network to perform PGNAA energy spectrum analysis based on Monte Carlo (MC) simulation. The main work is as follows: 
(1) complete the MC simulation of PGNAA spectral library and set the mass fraction of $\mathrm{Si}, \mathrm{Ca}$, and $\mathrm{Fe}$ element content range from 0.00 to 0.45 , the step size is 0.05 , and MCNP is used to simulate each sample. (2) The BP model of adaptive quantitative analysis of PGNAA energy spectrum was established. We calculated the peak areas of 8 characteristic gamma rays, which corresponded to 8 elements in 1000 samples and standard samples, respectively. (3) The feasibility of the quantitative analysis of the adaptive algorithm using 68 samples continuously is verified. The results show that the accuracy of using neural network to calculate the content of each element is significantly higher than MCLLS [8].

\subsection{Innovation in this article}

The main innovative work of this article includes the following aspects: (1) the various physiological indicators are expressed in a visual and quantitative way through tables, images, etc. At the same time, it is safe and non-invasive and can reflect the human body's nerves objectively to the greatest extent characteristics of muscle activity. (2) For the first time, a genetic algorithm based on BP neural network unconstrained optimization problem is proposed; algorithm steps and block diagram are given.

\section{Fatigue method of running sportswear based on BP neural network}

\subsection{Evaluation method of muscle fatigue in running}

The measurement method of bioelectrical signal sEMG (surface myoelectricity) is a comprehensive superposition of the electrical signals emitted by the relevant motor units during muscle contraction at the electrode in time and space, which can objectively and quantitatively reflect neuromuscular activities to a certain extent. sEMG's evaluation of muscle fatigue is mainly divided into two methods: time domain analysis and frequency domain analysis $[9,10]$.

(1) Time domain evaluation method. Time domain analysis refers to the characteristics of the amplitude changes of the EMG signal in the time dimension. Its indicators include RMS (root mean square amplitude), iEMG (integrated EMG), and other indicators such as MA (average amplitude) [11, 12]. RMS can reflect the average change characteristic of EMG signal in a period of time. A large number of studies believe that it is related to the recruitment of motor units and the synchronization of excitable rhythms $[13,14]$. Its definition is:

$$
R M S=\sqrt{\frac{1}{N} \sum_{i=1}^{N} x_{i}{ }^{2}}
$$

This represents the time series of EMG signal of length $N$. The change of EMG signal amplitude is affected by many factors, such as the number of sports potential recruitment, sports potential release frequency, action potential transmission speed, and the distance between muscle fiber and sensor; the strength of the muscle will also affect the RMS; the greater the muscle strength, the larger the RMS; these factors will affect the accuracy of the RMS $[15,16]$. iEMG is the sum of the area of the muscle electrical signal in the unit time after rectification and filtering [17]. It can characterize the strength and weakness of the myoelectric signal over time. It is important for fatigue evaluation. It is one of the means $[18,19]$. Its definition is: 


$$
X_{i E G M}=\frac{1}{N} \sum_{i=1}^{N}\left|x_{i}\right|
$$

Within a certain period of time, the more the unit's motor potential and the discharge amount of each sports unit, the larger the iEMG value, and the response to muscle fatigue is very specific. MA is commonly used to characterize the discharge level of muscles within a certain period of time, reflecting that the strength of EMG signal is related to the number of motor units involved and the degree of synchronization of discharge frequency [20, 21].

(2) Frequency domain evaluation method. Frequency analysis refers to the analysis of EMG signals from the frequency of signal changes. It has better stability than the time domain indicators of EMG signals. The frequency domain indicators are obtained by FFT (fast Fourier transform) [22]. The indexes of frequency domain analysis mainly include MF (median frequency) and MPF (average power frequency) [23].

MF refers to the intermediate value of the muscle contraction discharge frequency, which is mainly affected by the composition ratio of the fast muscle and the slow muscle of the muscle tissue. When the fast muscle is excited, the signal spectrum moves to a high frequency, and when the slow muscle is excited, the signal spectrum moves at a low frequency. Its definition is:

$$
M F=\frac{1}{2 M} \sum_{j=1}^{M} p_{j}
$$

The changes in the EMG signal spectrum during fatigue are related to factors such as muscle fiber conduction velocity and the duration of the motor unit potential waveform. As the degree of fatigue increases, the EMG signal spectrum will shift and the MF value will gradually decrease. MF is stable, has strong anti-interference ability, and has a wide range of load levels. It is widely used in the monitoring and analysis of surface EMG signals. MPF represents the frequency of the center of gravity of the power spectrum curve and is also a commonly used method in frequency domain analysis, but the response to time series changes is less consistent with MF. Therefore, only MF is selected as an indicator of frequency domain analysis.

\subsection{BP neural network training and testing}

A successful BP neural network model is not only reflected in the rationality of the neural network layer setting, but also in the practicality of solving the problem. Only the trained and tested network can meet the actual application requirements.

The structure of the BP neural network determines the nature and performance of the BP neural network. Its structure refers to the number of layers and the number of neurons in the BP neural network. The number of input layer and output layer neurons in the neural network is determined according to the actual problem itself, and the number of hidden layer neurons is variable. The determination of the structure of the BP neural network must first determine the input layer of the BP neural network according to the actual problem and the number of neurons in the output layer, and then determine the number of hidden layers and the number of hidden layer neurons. As long as the BP neural network contains enough hidden layers and hidden layer 
neurons, the BP neural network can approximate almost any function, but it may affect the performance of the BP neural network. With the increase of the number of hidden layers and neurons, the ability of the neural network to process information will be enhanced, but the transmission speed of the input signal from the input layer to the output layer will be slower, and the correction of the weights and thresholds will become more because of complexity; the speed of backpropagation becomes slower. It can be explained using the Occam's razor principle, that is, as long as there is a simpler network that works, do not use a more complicated network.

By collecting data from similar projects in the early stage for training, the weights are constantly adjusted to meet the error requirements of the BP neural network. Combined with the approximate degree of the test data results to verify the feasibility of the network, a more mature BP neural network is finally obtained. The flow of BP network work is shown in Fig. 1.

\subsection{Fatigue feature value extraction}

As the two main biological electrical signals of the human body, surface myoelectric signals and electrocardiographic signals contain a large amount of useful physiological information of the human body. The important task of fatigue detection technology is to extract effective and reliable characteristic indexes from the collected physiological signals for fatigue detection. The quality of feature extraction directly determines the accuracy of multi-class recognition. Therefore, it is necessary to select a feature index that represents the essence of fatigue and changes in feature differences. ECG signals are electrical signals that reflect heart activity, and can objectively reflect different physiological states of the human body. The human body is an organism with a complex structure and function. To evaluate the physiological state of the human body, a comprehensive analysis of multiple information parameters is required to realize the feature extraction evaluation method of multi-signal acquisition.

First, the time domain analysis method is used to extract the characteristics of the ECG signal and the EMG signal, and the ECG signal extracts the time domain indicators such as heart rate and heart rate variability. EMG signals are extracted from time-

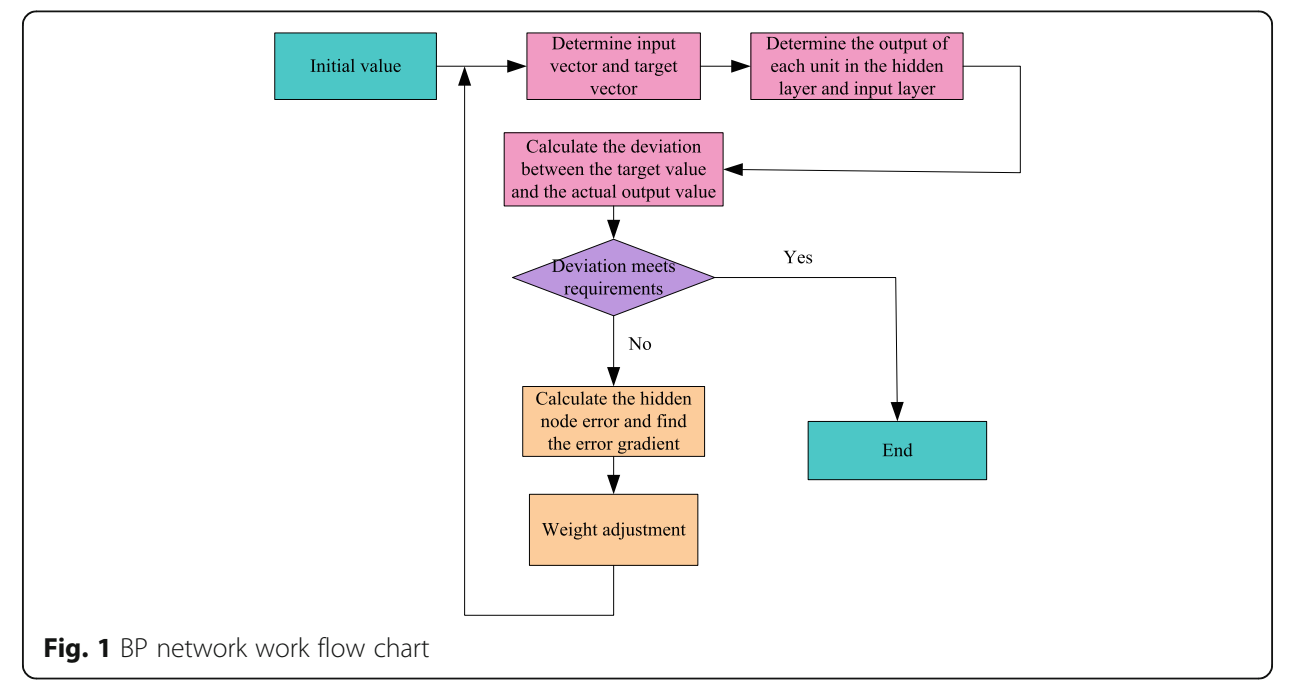


domain indicators such as root mean square (RMS), reshaping average (ARV), and integrated EMG (IEMG). The frequency domain analysis method is to convert the information in the time domain to the information in the frequency dimension through Fourier transform. The ECG signal extracts high-frequency power (HF, 0.15-0.4 Hz), low-frequency power (HF, 0.04-0.15 Hz), Frequency domain indicators such as the ratio of low frequency power to high frequency power (LF/HF), EMG signals are extracted from frequency domain indicators such as mean power frequency (Mean Power Frequency: MPF) and median frequency (Media Frequency: MF). Frequency domain analysis can reflect the frequency spectrum, power spectrum and other information of the signal in the frequency dimension.

This paper separately extracts features from the time domain and frequency domain, analyzes the changing trends of different characteristics of ECG and EMG signals under different fatigue states of the human body, and selects through experiments to change the features with large differences, good classification effect, and can reflect the movement state Substantial features are input as multiple classifiers. Using the multi-level structure and learning ability of Long-Short-Term Memory Neural Network (LSTM), these features are learned to find the inherent relationship between these features and the state of movement to find indicators with large differences for exercise fatigue judgment.

\subsection{BP neural network principle algorithm}

BP neural network (backpropagation neural network) is an artificial neural network based on BP algorithm, which uses BP algorithm to adjust the weight and threshold. In the 1980s, several different scholars developed backpropagation algorithms for training multilayer perceptrons. The backpropagation algorithms proposed by David Rumelhart and James McClelland are the most influential. It contains the two main processes of BP, namely the forward propagation of the working signal and the backward propagation of the error signal. The backward propagation of the error signal is to modify the weights and thresholds layer by layer from back to front, in order to make the actual output closer to the expected output.

Input $\mathrm{p}$ learning samples, which are represented by $x^{1}, x^{2}, \ldots x^{p}, \ldots$ After inputting the $p$ th sample to the network, the output $y_{j}^{p}(J=1,2, \ldots m)$ is obtained. Using the squared error function, the error $E_{p}$ of the $p$ th sample is obtained.

$$
E_{p}=\frac{1}{2} \sum_{j-1}^{m}\left(t_{j}^{p}-y_{j}^{p}\right)^{2}
$$

Use the cumulative error $\mathrm{BP}$ algorithm to adjust $w_{j k}$ to make the global error $\mathrm{E}$ smaller, that is

$$
\Delta w_{j k}=-n \frac{\partial E}{\partial w_{j k}}=-n \frac{\partial}{\partial w_{j k}}\left(\sum_{p-1}^{p} E_{p}\right)=\sum_{p-1}^{p}\left(-n \frac{\partial E_{p}}{\partial w_{j k}}\right)
$$

In the test, $n$ represents the learning rate. Define the error signal as: 


$$
\delta_{y j}=-\frac{\partial E_{p}}{\partial S_{j}}=-\frac{\partial E_{p}}{\partial y_{j}} \cdot \frac{\partial y_{j}}{\partial S_{j}}
$$

The first of them:

$$
\frac{\partial E_{p}}{\partial y_{j}}=\frac{\partial}{\partial y_{j}}\left[\frac{1}{2} \sum_{j-1}^{m}\left(t_{j}^{p}-y_{j}^{p}\right)^{2}\right]=-\sum_{j-1}^{m}\left(t_{j}^{p}-y_{j}^{p}\right)
$$

Second section:

$$
\frac{\partial y_{j}}{\partial S_{j}}=f_{2}{ }^{\prime}\left(S_{j}\right)
$$

The partial differential of the transfer function of the output layer, so:

$$
\delta_{y j}=\sum_{j-1}^{m}\left(t_{j}^{p}-y_{j}^{p}\right) f_{2}^{\prime}\left(S_{j}\right)
$$

From the chain theorem;

$$
\begin{aligned}
& \frac{\partial E_{p}}{\partial w_{j k}}=\frac{\partial E_{p}}{\partial S_{j}} \cdot \frac{\partial S_{j}}{\partial w_{j k}}=-\delta_{y j} z_{k} \\
& -\delta_{y j} z_{k}=-\sum_{j-1}^{m}\left(t_{j}^{p}-y_{j}^{p}\right) f_{2}^{\prime}\left(S_{j}\right) \cdot z_{k}
\end{aligned}
$$

Therefore, the weight adjustment formula of each neuron in the output layer is:

$$
\Delta w_{j k}=\sum_{p-1}^{p} \sum_{j-1}^{m} n\left(t_{j}^{p}-y_{j}^{p}\right) f_{2}^{\prime}\left(S_{j}\right) z_{k}
$$

(1) The working signal is propagated forward. The input signal enters from the input layer, enters the hidden layer neurons through synapses, and is transferred to the output layer after the transfer function calculation, and the output signal is calculated and output at the output layer. When the working signal is propagated in the forward direction, the weights and thresholds are fixed, and the state of each layer in the neural network is only related to the net output, weights, and thresholds of the previous layer. If the forward propagation obtains the desired output at the output layer, the learning ends, and the current weights and thresholds are retained.

(2) The error signal propagates in reverse. If the expected output is not obtained after the working signal is propagated in the forward direction, the error signal is calculated for backward propagation, and the difference between the actual output of the BP neural network and the expected output is calculated as the error signal. The output layer propagates layer by layer to the input layer. In this process, the weights and thresholds of this layer are modified every time a layer is propagated forward, thereby propagating forward to the input layer. This process is to make the result of the neural network closer to the expected result. After the forward propagation and the backward propagation, if the error still cannot meet the 
requirements, the process continues until the error meets the accuracy or meets other set end conditions such as the number of iterations.

\section{Running fatigue experiment based on BP neural network}

\subsection{Experimental data collection}

Twenty-five young healthy women with a height of $155-165 \mathrm{~cm}$ and a weight of 44-54 $\mathrm{kg}$ who love running are randomly selected from college students, and the body shape data is collected using the US non-contact 3D body scanner, as shown in Table 1. Fifteen women with lower body shape data (waist circumference, front waist height, back waist height, left waist height, right waist height, hip circumference) were selected as the test objects to ensure the accuracy of the leg dressing experiment. All subjects were required of the following: no abnormalities in cardiopulmonary function, familiar with experimental procedures and operating procedures, and, before the experiment, maintain good work and rest habits and a happy mood.

\subsection{Experimental samples}

According to market research, the seamless knitted sports shorts have become more popular for sports enthusiasts due to the special seamless knitting process, which makes the clothing more suitable for the human body and have better elasticity. At the same time, this topic is to find out the effect of clothing pressure on the fatigue of clothing and then to find the threshold of clothing fatigue. In order to simplify and minimize the interference of unnecessary factors, this experiment chose seamless sports shorts woven by Zhejiang Bangjie Knitting Digital Co., Ltd.

\subsection{Experimental process}

1) This experiment was conducted in a constant temperature and humidity climate room with a room temperature of $(22 \pm 2){ }^{\circ} \mathrm{C}$ and a relative humidity of $(65 \pm 2) \%$, in order to ensure the accuracy of the experimental results. After entering the laboratory, the formal experiment began 30 min later.

2) Participants wore seamless knitted tight sports shorts with three specifications of 155/64A, 160/68A, and 165/72A in turn and performed a 30-s running in situ exercise. In order to better match the real running process, the first after accelerating, the motion mode of uniform speed and then deceleration, and the duration of

Table 1 Descriptive statistics of test subject data

\begin{tabular}{lllllll}
\hline & $\boldsymbol{N}$ & Minimum & Maximum & Mean & Standard deviation & Variance \\
\hline Height & 15 & 155.2 & 164.3 & 160.4 & 1.88 & 3.62 \\
Body weight & 15 & 44.52 & 53.6 & 50.2 & 2.14 & 4.56 \\
Waistline & 15 & 66.28 & 74.65 & 71.24 & 2.42 & 5.5 \\
Front waist high & 15 & 88.75 & 89.34 & 94.25 & 2.66 & 6.44 \\
Waist height & 15 & 89.63 & 95.28 & 92.34 & 2.92 & 7.38 \\
Left waist high & 15 & 85.24 & 88.95 & 91.27 & 2.18 & 5.32 \\
Right waist high & 15 & 85.24 & 88.95 & 91.27 & 3.44 & 3.26 \\
Hips & 15 & 87.14 & 98.62 & 93.24 & 3.75 & 4.21 \\
\hline
\end{tabular}


acceleration, uniform speed, and deceleration process are $6 \mathrm{~s}, 18 \mathrm{~s}$, and $6 \mathrm{~s}$ in sequence.

3) The same test subject has a 10-min interval between two tests. The pliance multipurpose sensor system produced by the German company Novel is used to measure the clothing pressure value of the five leg test points during the test run. The front-end sensor sheet is attached to the test point, and then the clothing is put on to cover it. The thickness of the induction zone is only $0.2 \mathrm{~mm}$, the diameter is $9.52 \mathrm{~mm}$, and the force range can reach up to $60 \mathrm{kpa}$. Because the pressure value of the clothing is small, the pressure test range is set to $0 \sim 24 \mathrm{kpa}$. The plianceX test software that comes with the system has Window operation function.

4) The dynamic pressure data collected on the pressure sensor can be transmitted to the computer in real time through the dongle and wireless transmitter, the experimental data can be output, and the average value of the pressure value of each test point is taken as the clothing pressure value of each part.

\section{Influencing factors of running sportswear fatigue based on BP neural network}

4.1 Running fatigue analysis based on BP neural network

In the frequency domain analysis of surface EMG signals, fast Fourier transform (FFT) is performed on the EMG signals in the time domain to obtain power spectrum and spectrum change information in the frequency dimension of the surface EMG signals. Through the exercise fatigue exhaustion experiment, the surface myoelectric signals of the leg muscles, rectus femoris and medial femoral muscles, were collected during the experiment, the original EMG signals were filtered, and the time and frequency domain feature indicators were extracted: shaping. The average value (ARV), integrated myoelectric value (IEMG), root mean square value (RMS), and average power frequency (MPF: frequency power mean, median frequency (MF: frequency media)) for sports fatigue correlation analysis are used. The indicators of average power frequency (MPF: frequency power mean) and median frequency (MF: frequency media) describe the characteristics of EMG signals in the frequency domain. The results are shown in Table 2. To facilitate image representation, the data size is normalized to less than 100 .

According to the data in Table 2 and Table 3, the histograms of different muscle indicators under various exercise states are obtained, as can be seen in Figs. 2 and 3. Under different levels of sports fatigue, the surface EMG signals of the two muscles have various characteristic indicators. There are certain changes. The time domain indexes RMS, ARV, and IEMG deepen with increasing exercise time, and the

Table 2 Straight femoral electromyography indexes of different exercise states

\begin{tabular}{lllll}
\hline Muscle name & Index & \multicolumn{3}{l}{ Movement state } \\
\cline { 3 - 5 } & & Very relaxed & A bit tired & Very tired \\
\hline Rectus femoris & ARV & $4.85 \pm 0.34$ & $6.21 \pm 0.37$ & $7.32 \pm 0.16$ \\
& IEMG & $13.84 \pm 0.73$ & $15.78 \pm 0.85$ & $19.74 \pm 1.47$ \\
& RMS & $16.67 \pm 2.35$ & $23.76 \pm 2.16$ & $34.37 \pm 2.12$ \\
& MPF & $65.21 \pm 2.54$ & $56.42 \pm 2.42$ & $56.23 \pm 2.37$ \\
& MF & $42.48 \pm 1.74$ & $39.75 \pm 1.52$ & $37.68 \pm 1.27$ \\
\hline
\end{tabular}


Table 3 Medial femoral electromyography indexes of different exercise states

\begin{tabular}{lllll}
\hline Muscle name & Index & \multicolumn{3}{l}{ Movement state } \\
\cline { 3 - 4 } & & Very relaxed & A bit tired & Very tired \\
\hline Medial femoral muscle & ARV & $16.84 \pm 0.34$ & $17.39 \pm 0.29$ & $21.62 \pm 0.79$ \\
& IEMG & $18.66 \pm 1.36$ & $23.16 \pm 1.41$ & $35.17 \pm 2.51$ \\
& RMS & $18.29 \pm 2.92$ & $36.53 \pm 3.41$ & $58.16 \pm 3.52$ \\
& MPF & $58.24 \pm 4.13$ & $57.35 \pm 4.57$ & $54.79 \pm 3.64$ \\
& MF & $36.24 \pm 1.38$ & $27.68 \pm 1.42$ & $26.39 \pm 1.49$ \\
\hline
\end{tabular}

characteristic index values gradually increase. The characteristic index RMS with the greatest degree of change is used as the time domain index for judging muscle fatigue. The frequency domain feature indexes MPF and MF gradually decrease with the deepening of sports fatigue. The feature index MPF has better variation difference and stability compared to MF. The feature index MPF is used as the frequency domain index for human sports fatigue assessment. Analysis of the measured index of the EMG signals on the surface of the two muscles of the leg shows that the characteristic values of the EMG signals of different muscles in different degrees of fatigue will change accordingly, and the change trend of each characteristic value is the same, indicating that the measured EMG signals of the rectus peroneus and medial femoris muscles can objectively reflect the changes of human sports fatigue. The frequency and time domain index values of the lateral femoral muscle are large and the difference is significant, and it is more difficult to objectively reflect human sports fatigue. Therefore, in this paper, the frequency domain index MPF and the time domain index RMS of the lower extremity lateral femoral muscle are finally used for the exercise fatigue classification experiment.

\subsection{Comparison of dress fatigue assessment results based on BP neural network prediction model}

The BP neural network trained the overall training data of the running people. The AUC reached 0.942 , the sensitivity and specificity were $69 \%$ and $86 \%$, and the accuracy

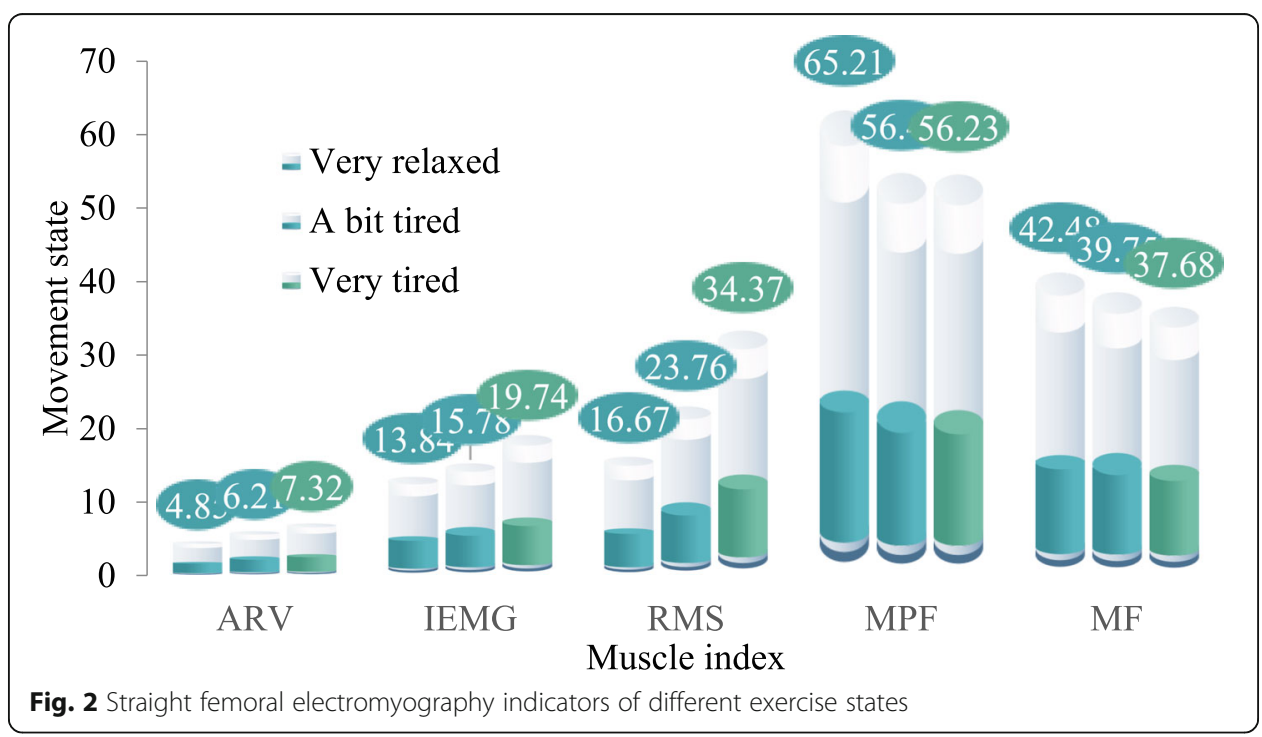




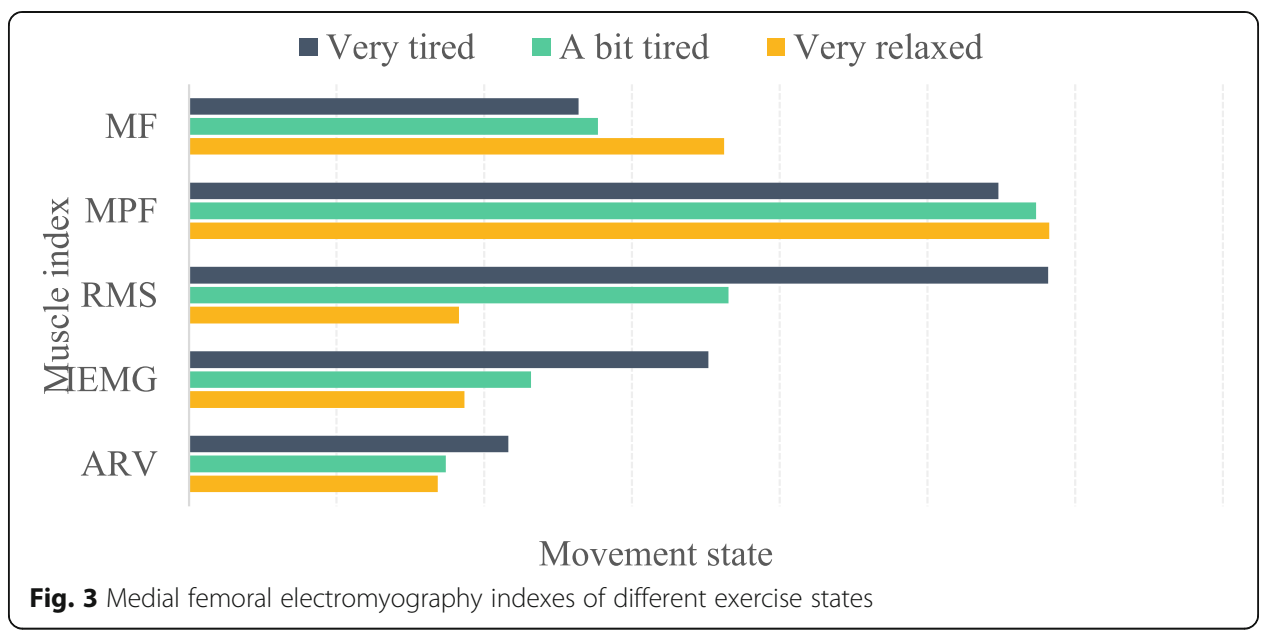

reached $100 \%$. It is far higher than the prediction results of OSTA in the middle-aged and elderly $(P<0.05)$; the sensitivity, specificity, and accuracy are $86 \%, 20 \%$, and $90 \%$ respectively. Use the trained model to predict the verification group. The AUC of the verification group is 0.6 , the sensitivity and specificity are $60 \%$ and $73 \%$, and the accuracy is $68.5 \%$. The verification result is significantly lower than the training group results, and the difference between the prediction results and the OSTA in the verification group is not statistically significant $(P=0.562)$. The performance of OSTA in the verification group is even better, with an AUC of 0.756 , sensitivity and specificity of $72 \%$ and $19 \%$, and accuracy of $65 \%$, as shown in the Table 4 and Fig. 4.

\subsection{Analysis of the effect of running sportswear fatigue on blood circulation system}

When wearing tight-fitting sports pants for vertical jumping, the muscles wrapped in the lower extremities are significantly reduced in both lateral and vertical muscle vibrations. Another study pointed out that in running sports, muscle vibration decreases and muscle displacement decreases when wearing tight sports pants. The reduction of muscle vibration can not only prevent muscle or tendon damage but also save unnecessary muscle coordination and reduce energy consumption. The main role of blood circulation in muscle activity is to supply blood to provide oxygen and nutrients and, at the same time, transport waste gas $\left(\mathrm{CO}_{2}\right)$ and other products generated in the metabolic process to the corresponding organs to be discharged from the body. When the human body is in motion, stronger blood circulation is needed to meet the strong metabolic needs. Appropriate tight compression will have a certain promoting effect on the body's blood circulation function, as shown in Fig. 5.

Table 4 ROC curve comparison between OSTA and BP neural network models

\begin{tabular}{|c|c|c|c|c|c|c|c|}
\hline \multicolumn{2}{|c|}{ ROC index comparison } & \multirow{2}{*}{$\frac{\text { AUC }}{0.586}$} & \multirow{2}{*}{$\frac{\text { SE }}{89.4}$} & \multirow{2}{*}{$\frac{\text { SP }}{18.4}$} & \multirow{2}{*}{$\frac{\text { ACC }}{43.9}$} & \multirow{2}{*}{$\frac{Z \text { value }}{9.63}$} & \multirow{2}{*}{$\begin{array}{l}P \\
<0.002\end{array}$} \\
\hline Training group & OSTA & & & & & & \\
\hline & BP neural network & 0.942 & 69.4 & 86.5 & 93.4 & & \\
\hline \multirow[t]{2}{*}{ Verification group } & OSTA & 0.756 & 93.8 & 19.5 & 48 & 1.63 & 0.562 \\
\hline & BP neural network & 0.679 & 59.6 & 72 & 65.3 & & \\
\hline
\end{tabular}




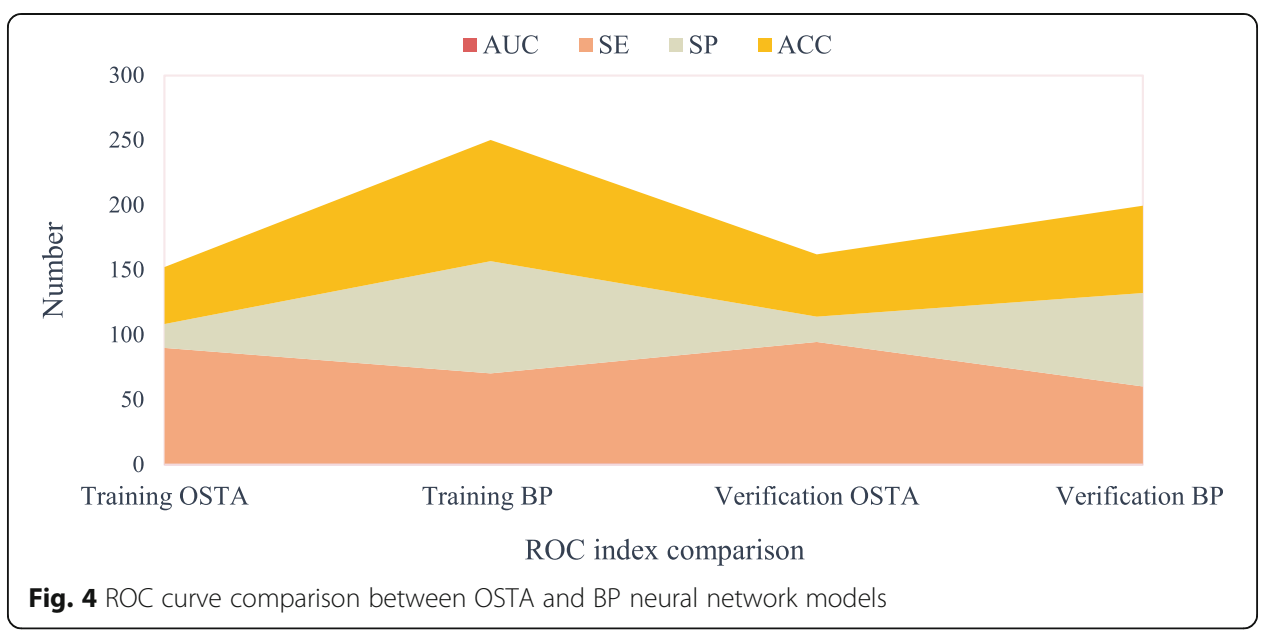

The data in Fig. 5 indicate that applying a gradually decreasing gradient pressure from the ankle to the lower limb can promote venous return. In clinical medicine, doctors wear a gradient compression stocking to prevent and treat lower limb venous thrombosis, varicose veins, and edema. In the field of sports, some professional sports pantyhose began to adopt this gradient pressure design to help athletes accelerate the pulse flow of the lower limbs during training or competition.

\subsection{Discussion}

The research value of this topic lies in the following: revealing the influence mechanism of dress fatigue from the fields of motor neurology, ergonomics, physiology, and clothing engineering and providing some theoretical and technical references for manufacturers of women's running shorts. When designing and developing sports shorts, apparel companies can control the clothing pressure generated by different parts to minimize wear fatigue and open up a new way for the textile industry to better weave high-performance tight-fitting sportswear that relieves wear fatigue. Of course, there are some shortcomings, as follows:

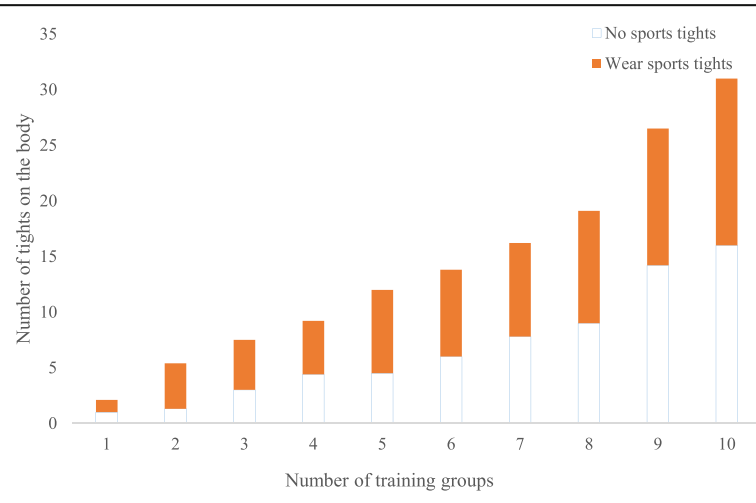

Fig. 5 Number of stresses in sports tights during running 
1) The experimental samples only carried out three specifications of seamless knitted tight-fitting sports shorts and did not use more types of samples and exercise intensity for experiments. The research may not be comprehensive enough, and further discussion is needed. In future research, it can be extended to different fabrics, organizational structures, and interweaving ratios, so that the conclusions are more scientific and comprehensive, and the coverage rate is wider.

2) For the collection of physiological data, this topic only uses a limited number of test indicators and only measures the impact of the athlete's blood circulatory system. In future research, more compact instruments can be used while continuing to expand the test indicators.

3) The object of this research is young female college students in school, who are good in all aspects of physical fitness. In future research, research can be carried out for women of different ages.

4) Part of the test site of the female lower limbs was selected for the test site of this experiment, and the calf and upper limbs were not discussed. In future research, the test site can be continued to be expanded to make a more comprehensive and in-depth study of the mechanism of clothing fatigue and research and develop more high-performance sportswear.

\section{Results}

This article is based on the BP neural network. The wearing of tight sports pants has a positive effect on muscle activity. The tight sports pants (GCT) produce compression which can reduce muscle vibration, thereby reducing energy consumption, making myoelectric amplitude and average electromyography (aEMG) smaller than when wearing loose track pants; compression can also promote sympathetic nerve activity and increase nerve excitability, thus changing the way muscle fibers are recruited; at the same time, tight compression can also enhance the proprioception of muscles and joints.

In this paper, the BP neural network is used to distribute the weights of a single model autonomously, and the prediction results of the SVR and ELM single models are nonlinearly combined. At the same time, the influencing factors of the heat load are also taken into account. The prediction accuracy of the combined method is improved compared with the single method of PSO-SVR and ELM. It can be proved that the combined method can combine the advantages of a single prediction model and improve the prediction accuracy.

In this study, running sports apparel evaluation tools were used to screen the research objects. Given appropriate cutoff values, commonly used tools have high sensitivity, but low specificity and accuracy. Through training, the BP neural network balances sensitivity and specificity, improves accuracy, and has the same screening ability as common running exercise evaluation tools.

\footnotetext{
Abbreviations

PSO: Particle swarm optimization; PGNAA: Prompt gamma neutron activation analysis; MC: Monte Carlo; SEMG: Surface electromyography; EMG: Electromyography; RMS: Root mean square; IEMG: Integrated electromyography; MA: Average amplitude; FFT: Fast Fourier transform; MF: Median frequency; MPF: Frequency power mean; ARV: Average value; HF: High-frequency power; LF: Low-frequency power; LF/HF: Low frequency power to high frequency power
} 


\section{Authors' contributions}

Xiaoli Liu: writing—original draft, validation, methodology. Zhibin Li: conceptualization, formal analysis, data curation. The authors read and approved the final manuscript.

\section{Authors' information}

Xiaoli Liu was born in Zhongjiang, Sichuan, P.R. China, in 1974. She received the Master's degree from Shanghai University of Sport, P.R. China. Now, she works in the Dept. of Physical Education, Shanghai University of Electric Power; her research interests include physical education teaching and training.

Zhibin Li was born in Taiyuan, Shanxi P.R. China, in 1974. He received the Doctor of philosophy from University of Shanghai for Science and Technology, P.R. China. Now, he studies in the College of Automation Engineering, Shanghai University of Electric Power. His research interests include advanced measurement technology, computational intelligence, and fault diagnosis.

\section{Funding}

The article was not funded.

\section{Availability of data and materials}

Articles will not share data. Data sharing does not apply here, because no data sets were generated or analyzed during the current study period.

\section{Declarations}

\section{Ethics approval and consent to participate}

The article has been ethically approved and approved.

\section{Consent for publication}

All presentations of the case report have been agreed for publication.

\section{Competing interests}

The authors declare that they have no competing interests.

\section{Author details}

${ }^{1}$ Department of Physical Education, Shanghai University of Electric Power, Shanghai 200090, China. ${ }^{2}$ College of Automation Engineering, Shanghai University of Electric Power, Shanghai 200090, China.

Received: 29 May 2021 Accepted: 26 July 2021

Published online: 06 August 2021

\section{References}

1. M. Lindquist Liljeqvist, R. Hultgren, T.C. Gasser, et al., Volume growth of abdominal aortic aneurysms correlates with baseline volume and increasing finite element analysis-derived rupture risk. J. Vasc. Surg. 63(6), 1434-1442 (2016) e3

2. S.O. Olajide, B.D. Arhatari, Progress on interacting fatigue, creep \& hysteretic heating in polymer adhesively bonded composite joints. Int. J. Fat. 98(May), 68-80 (2017)

3. C. Chen, X. Chen, S. Guo, Experimental study on acoustic emission characteristic of fatigue crack growth of selfcompacting concrete. Struct. Control Health Monit. 26(4), e2332.1-e2332.17 (2019)

4. I.G. Goryacheva, F.I. Stepanov, E.V. Torskaya, Fatigue wear modeling of elastomers. Physical Mesomechanics 22(1), 65-72 (2019)

5. M. Giandolini, G. Vernillo, P. Samozino, et al., Fatique associated with prolonged graded running. Eur. J. Appl. Physiol. 116(10), 1859-1873 (2016)

6. B. Wu, S. Han, J. Xiao, et al., Error compensation based on BP neural network for airborne laser ranging. Optik Int. J. Light \& Electron Optics 127(8), 4083-4088 (2016)

7. Jidong Wang, Kaijie Fang, Wenjie Pang. Wind power interval prediction based on improved PSO and BP neural network J. Electric. Eng. Technol., 2017, 12(3):989-995.

8. X. Tongle, W. Yingbo, C. Kang, Tailings saturation line prediction based on genetic algorithm and BP neural network. J. Intelligent Fuzzy Syst. 30(4), 1947-1955 (2016)

9. A. Namura, H. Tanimoto, F. Urakawa, et al., Rail surface fatigue assessment using numerical simulation of the concurrence of short crack propagation and wear. Quarterly Report of Rtri 57(4), 311-317 (2016)

10. J. Song, X. Liu, G. Zhao, et al., Effect of surface roughness and reciprocating time on the tribological properties of the polyimide composites. Polymer Eng. Sci. 59(3), 483-489 (2019)

11. Wang J , Zhou C. Finite element analysis of the wear fatigue of rails with gradient structures. Materials Letters. 2018 231(NOV.15):35-37

12. Kim $Y$, Lee $K$, Yong-Hyun Cho.... Fatigue safety evaluation of newly developed contact wire for eco-friendly high speed electric railway system considering wear. Int. J. Precision Eng. Manufacturing Green Technol., 2016, 3(4):353-358

13. G. Zhang, R. Ren, T. Cong, et al., Test study on wear resistance of domestic wheel for $250 \mathrm{~km} \cdot \mathrm{h}-1$ high speed EMU. Zhongguo Tiedao Kexue China Railway Sci. 38(1), 117-122 (2017)

14. H. Lee, Y. Lee, Evaluation of wear satisfaction and subjective fatigue for developing a baby carrier. Korean J. Hum. Ecol. 26(4), 313-326 (2017)

15. Senthil KUMAR Muniyandi. Investigation of surface coating on wear,corrosion and fatigue behavior of steel-a review. Adv. Natural Appl. Sci., 2016, 10(7):319-325.

16. P. Droyner, Gas pipeline structural fatigue life prediction based on vibration signals analysis. J. Vibroeng. 18(8), 52395251 (2016) 
17. M. Zhou, Y. Long, W. Zhang, et al., Adaptive genetic algorithm-aided neural network with channel state information tensor decomposition for indoor localization. IEEE Trans. Evol. Comput. (2021). https://doi.org/10.1109/TEVC.2021.3 085906

18. Juanjuan $R$, Xiao $L$, Rongshan $Y$, et al. Criteria for repairing damages of CA mortar for prefabricated framework-type slab track. Construct. Build Mater., 2016, 110(may1):300-311.

19. D'Angelo L, Nussbaumer A. Estimation of fatique S-N curves of welded joints using advanced probabilistic approach. Int. J. Fat., 2017, 97(APR.):98-113.

20. D. Spriestersbach, P. Grad, E. Kerscher, Threshold values for very high cycle fatigue failure of high-strength steels. Fatigue Fract. Eng. Mater. Struct. 40(11), 1708-1717 (2017)

21. Liu Y M, Wang L, Chen G, et al. Investigation on ratcheting-fatigue behavior and damage mechanism of $\mathrm{GH} 4169$ at $650^{\circ} \mathrm{C}$. Materials Ence. Eng., 2019, 743(JAN.16):314-321.

22. M. Zhou, Y. Li, M.J. Tahir, et al., Integrated statistical test of signal distributions and access point contributions for Wi-Fi indoor localization. IEEE Trans. Vehicular Technol. 70(5), 5057-5070 (2021)

23. W. Wronicz, Comparison of residual stress state on sheets faying surface after standard and NACA riveting-numerical approach. Nephron Clin. Pract. 2016(8), 116-126 (2016)

\section{Publisher's Note}

Springer Nature remains neutral with regard to jurisdictional claims in published maps and institutional affiliations.

Submit your manuscript to a SpringerOpen ${ }^{\odot}$ journal and benefit from:

- Convenient online submission

- Rigorous peer review

- Open access: articles freely available online

- High visibility within the field

- Retaining the copyright to your article

Submit your next manuscript at $\boldsymbol{\nabla}$ springeropen.com 